\title{
Unconscious, stimulus-dependent priming and conscious, percept-dependent priming with chromatic stimuli
}

\author{
BRUNO G. BREITMEYER \\ University of Houston, Houston, Texas \\ Tony Ro \\ Rice University, Houston, Texas \\ AND \\ Haluk ÖĞMen ANd STeven Todd \\ University of Houston, Houston, Texas
}

\begin{abstract}
Using metacontrast masking to suppress the conscious registration of a prime stimulus, Breitmeyer, Ro, and Singhal (2004) showed that color priming produced by a masked prime disk occurs at unconscious stimulusdependent rather than at percept-dependent levels of visual processing. The current set of experiments compares this type of unconscious stimulus-dependent priming to conscious priming produced by a prime that, in two separate ways, is rendered visible and thus activates percept-dependent visual processes. The results indicate that while the masked prime again acts at a stimulus-dependent level of processing, the unmasked, visible primes additionally act at a later percept-dependent level of processing.
\end{abstract}

During the past decade, the distinction between unconscious and conscious information processing and the types and levels of brain activity corresponding to them have been major topics of interest among cognitive psychologists and neuroscientists (Crick \& Koch, 1998; de Gelder, de Haan, \& Heywood, 2001; Kihlstrom, 1996; Kreiman, Fried, \& Koch, 2002; Leopold \& Logothetis, 1996; Metzinger, 2000; Milner \& Goodale, 1995; Weiskrantz, 1997; Zeki, 1997). An effective way to investigate differences between the two types of processing is to use stimuli whose attributes are suppressed from conscious registration by visual masks, without, however, suppressing information that can be made available to systems that rely on unconsciously processed information (Ansorge, Klotz, \& Neumann, 1998; Dolan, 2002; Esteves, Parra, Dimberg, \& Ohman, 1994; Klotz \& Wolff, 1995; Morris \& Dolan, 2001, Neumann \& Klotz, 1994). For example, when a masked prime and a following mask have congruent colors, such as a green prime followed by a green mask, choice reaction times (CRTs) to the mask color are shorter than when they have incongruent colors, such as a green prime followed by a red mask (Schmidt, 2000, 2002) or a blue mask (Breitmeyer, Öğmen, \& Chen, 2004; Breitmeyer, Ro, \& Singhal, 2004). We call such unconscious processing of the prime stimulus-dependent, since it depends only on the presence (but not the perceptual state: invisible or visible) of the prime stimulus. On the other hand, a processing level that is activated only if a prime stimulus is perceived can be characterized as percept-dependent.

Regarding chromatic stimuli, we take their wavelength composition to be an objectively specifiable physical property, regardless of whether they are perceived or not. On the other hand we take color to refer to one of the several consciously perceived attributes of the chromatic stimuli. Using the CRT paradigm with metacontrast masking to render a prime stimulus invisible, Breitmeyer, Ro, and Singhal (2004) found that the unconscious chromatic priming produced by a masked prime was consistent with an early, wavelength- or stimulus-dependent level of visual processing rather than a later, color- or percept-dependent one. In that study, white as well as desaturated blue or green prime disks were followed at an optimal metacontrast stimulus onset asynchrony (SOA) of $42 \mathrm{msec}$ by either a desaturated blue or green mask ring surrounding the primes. CRTs to a blue mask following a white prime were significantly slower than CRTs to a blue mask following a blue prime. In contrast, CRTs to a green mask following a white prime were not significantly different from CRTs to a green mask following a green prime. That is, the white prime had effects similar to those of the green prime but different from those of the blue prime. Moreover, control

B. G. Breitmeyer, brunob@uh.edu 


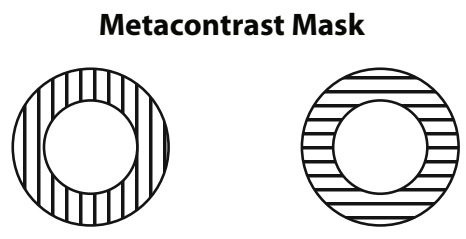

Prime/Prime

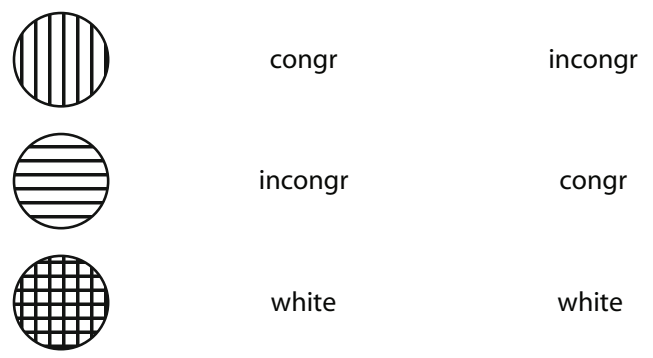

Figure 1. Schematic representation of the combinations of disk primes and mask masks. Horizontal, vertical, and crosshatched patterns designate blue, green, and white colors, respectively. In congruent ("congr") and incongruent ("incongr") trials, blue and green primes were presented with either blue or green annuli. In addition, white primes were paired with blue and green annuli.

experiments, in which the prime disks (presented without the mask) were clearly visible, indicated that the white disk perceptually resembled the blue one more than the green one. The similarity of the priming effect of the suppressed white disk to that of the suppressed green prime cannot, therefore, be based on the perceptual color properties of the disks. Consequently, the effect is most likely due to preperceptual, stimulus-dependent levels of visual processing.

Priming also occurs when the SOA between the prime and mask varies over a range of values that presumably includes - apart from the SOA at which complete masking occurs - SOAs at which the prime might be partially or even totally visible (Schmidt, 2002; Vorberg, Mattler, Heinecke, Schmidt, \& Schwarzbach, 2003). This raises the interesting question about different types and levels of unconscious and conscious color priming. In the following experiments, we address this question by comparing color priming with fully suppressed primes to priming when the primes are rendered visible. Since color priming occurs at wavelength-dependent levels when the primes are fully masked (Breitmeyer, Ro, \& Singhal, 2004), we, like Breitmeyer, Öğmen, and Chen (2004), expected to replicate the stimulus-dependent nature of unconscious color priming. However, when the primes are not masked and thus are visible, they must be processed up to a perceptdependent level. This raises the possibility that perceived primes correspondingly produce priming at later, perceptdependent rather than early, stimulus-dependent levels of processing.

\section{EXPERIMENT 1}

To render the prime invisible, we first determined for two of the authors (BB and ST) the SOA at which the prime was optimally masked. This SOA turned out to be $53 \mathrm{msec}$. Primes consisted of a 14-min-arc disk that could be a desaturated blue, a desaturated green or a white color. The mask consisted of a desaturated blue or green ring, with inner and outer diameters of 14 and $21 \mathrm{~min}$ arc, respectively. Consequently, the prime-mask contour separation was 0 min arc, referred to as the $0 / 53$ condition. Relative to this baseline invisible condition, we rendered primes visible by presenting the prime and mask at the optimal masking SOA of $53 \mathrm{msec}$; however, here the mask had an inner and outer diameter of 17.5 and $24.5 \mathrm{~min}$ arc, respectively, introducing a prime-mask contour separation of $3.5 \mathrm{~min}$ arc. This constitutes the $3.5 / 53$ condition. With foveally centered stimuli, this small contour separation weakens the masking effect sufficiently to render the primes visible (Breitmeyer, 1984, p. 109). We expected the $0 / 53$ condition to replicate our prior findings, showing that the white prime acted as an unconscious, wavelength-dependent prime by again being more similar to the green than to the blue prime. In contrast, we expected that the $3.5 / 53$ conditions would yield priming effects of the white prime disk consistent with its activating percept-dependent mechanisms.

\section{Method}

Participants. Seventeen students with no knowledge of the purpose of the experiment were selected from the University of Houston undergraduate student population and served as observers, having given prior voluntary informed consent to their participation. All observers had normal color vision.

Stimuli and Apparatus. All visual displays, generated by a Macintosh IIci microcomputer driving a Spectrum $/ 8$ graphics card, were presented on a 19 in. Trinitron high-resolution color monitor and were viewed at a distance of $57 \mathrm{~cm}$. At that distance the display dimensions of the monitor were $29^{\circ} \times 22^{\circ}$. The stimuli consisted of prime disks and mask annuli with dimensions as described above. The fixation stimulus consisted of two black line segments aligned along the horizontal meridian, each $3.5 \mathrm{~min}$ arc $\times 35 \mathrm{~min}$ arc, separated by 105 min arc and two black line segments aligned along the vertical meridian, each $35 \mathrm{~min}$ arc $\times 3.5 \mathrm{~min}$ arc, also separated by $105 \mathrm{~min}$ arc. Observers were asked to fixate on the imaginary intersection point provided by the vertically and horizontally notional lines. The stimuli, falling at the center of this notional cross, were presented on the color monitor with a refresh rate of $75 \mathrm{~Hz}$ (13.3 msec per frame). The disks were presented for one frame $(13.3 \mathrm{msec})$ and the annuli for two frames $(26.7 \mathrm{msec})$ at an optimal metacontrast SOA of $53.3 \mathrm{msec}$. The white background of the screen was set at a luminance of $6.0 \mathrm{~cd} / \mathrm{m}^{2}$. For the purposes of the present experiments it is necessary to use heterochromatic target and mask stimuli that yield strong metacontrast suppression of the target while still giving rise to chromatically specific priming effects. To meet these two criteria, we used chromatic stimuli that appeared equally desaturated. Our prior unpublished observations of heterochromatic metacontrast indicated that the visibility of target colors depended on their saturation. Specifically, while a green target was fairly readily suppressed by a saturated blue (or red) metacontrast mask, the reverse was not true. By desaturating the blue (or red) targets we were more readily able to suppress their visibility by a green metacontrast mask. For this reason, the desaturated blue stimuli were generated by adding a uniform-white increment of $7.5 \mathrm{~cd} / \mathrm{m}^{2}$, on which in turn was added a luminance increment of $2.4 \mathrm{~cd} / \mathrm{m}^{2}$ of the blue phosphor. The desaturated green was generated by adding as nearly as possible $9.9 \mathrm{~cd} / \mathrm{m}^{2}$ of the green phosphor only. The saturation values for the green and blue stimuli were nearly equal at $30.4 \%$ and $31.2 \%$, respectively. The white disk was generated by adding as nearly as possible a uniform white increment of $9.9 \mathrm{~cd} / \mathrm{m}^{2}$. Hence 
Table 1

CIE $(x, y)$ Coordinates and Luminances of the White Background and the Three Primes

\begin{tabular}{lccc}
\hline & \multicolumn{2}{c}{ Coordinate } & Luminance \\
\cline { 2 - 3 } & $x$ & $y$ & $\left(\mathrm{~cd} / \mathrm{m}^{2}\right)$ \\
\hline White background & 0.281 & 0.299 & 6.0 \\
White prime & 0.281 & 0.299 & 15.9 \\
Green prime & 0.281 & 0.339 & 15.9 \\
Blue prime & 0.258 & 0.258 & 15.9 \\
\hline
\end{tabular}

Table 2

Cone Contrasts for Each of the Three Primes

\begin{tabular}{cccc}
\hline & \multicolumn{3}{c}{ Cone Type } \\
\cline { 2 - 4 } Prime & LW & MW & SW \\
\hline White & 0.623 & 0.623 & 0.623 \\
Green & 0.618 & 0.631 & 0.527 \\
Blue & 0.619 & 0.629 & 0.717 \\
\hline
\end{tabular}

all primes had a luminance of $15.9 \mathrm{~cd} / \mathrm{m}^{2}$. Table 1 lists the CIE $(x, y)$ coordinates for the white background and the white, green, and blue stimuli; Table 2 lists the cone contrasts for each of the three primes (white, blue, green).

Procedure. Each observer participated in four 126-trial blocks, each lasting about 15 min with brief rest periods in between. Two of the blocks were devoted to measuring CRTs to the mask color, with one block for each of the two prime visibility conditions: 0/53 (prime invisible) and 3.5/53 (primes visible). In the CRT trials, the observer binocularly fixated the center of the screen; and a disk, presented for $13 \mathrm{msec}$, was followed by a 40-msec blank screen, which in turn was followed by a $27-\mathrm{msec}$ mask. The observer was instructed to depress as quickly and as accurately as possible one of two response keys to indicate whether the mask was blue or green. The intertrial interval was $1.5 \mathrm{sec}$. Pairings of target and mask colors were randomized across trials, subject to the restriction that an equal number of trials was devoted to each of the mask-color and prime-color pairings. This yielded 42 congruent ( 21 for blue-blue and 21 for green-green), 42 incongruent (21 green-blue and 21 blue-green), and 42 white ( 21 white-blue and 21 white-green) prime--mask pairings. For each pairing, mean RTs were computed for each observer. These values were in turn averaged to obtain grand means for each of the combinations of mask color, prime color, and congruency condition. Using the same general procedure, a third trial block of 126 trials was used to measure the visibility and perceptual confusability of the primes in the $0 / 53$ condition, and a fourth block to measure the same in the $3.5 / 53$ conditions. Here observers were asked to indicate, by pressing one of three response keys, the perceived color of the prime disks. Observers were given 3,000 msec to make their response and were asked to guess if they were not sure of the perceived color. These results allowed computation of the mean number of correct color identifications as well as perceived color confusions among the three prime disks. The order of the four trial blocks was randomized across the observers.

\section{Results and Discussion}

Of the 17 observers, 3 obtained correct prime-color identification proportions statistically above the chance guessing level of .333 (binomial test), indicating that for them the 53-msec SOA did not yield optimal masking. For that reason, their data were not included in the analyses described below. For those results included in the analysis of the CRT data of both the $0 / 53$ and the $3.5 / 53$ conditions, response errors (e.g., responding "green" when a blue mask was presented) averaged less than $3.1 \%$.

Mask CRTs for invisible primes (0/53). As in prior experiments (Breitmeyer, Öğmen, \& Singhal, 2004; Breitmeyer, Ro, \& Chen, 2004), the results showed that the white disk did not act as a neutral prime; rather, its effect depended on the color of the mask. A 2 (mask color) $\times 3$ (congruency) repeated measures ANOVA revealed significant main effects of congruency $[F(2,26)=12.16$, $p<.001]$ and mask color $[F(1,13)=12.74, p<.003]$, as well as a significant interaction effect $[F(2,26)=10.62$, $p<.001]$. Regarding the main effect, Figure 2A shows

\section{0/53 (Prime Invisible)}
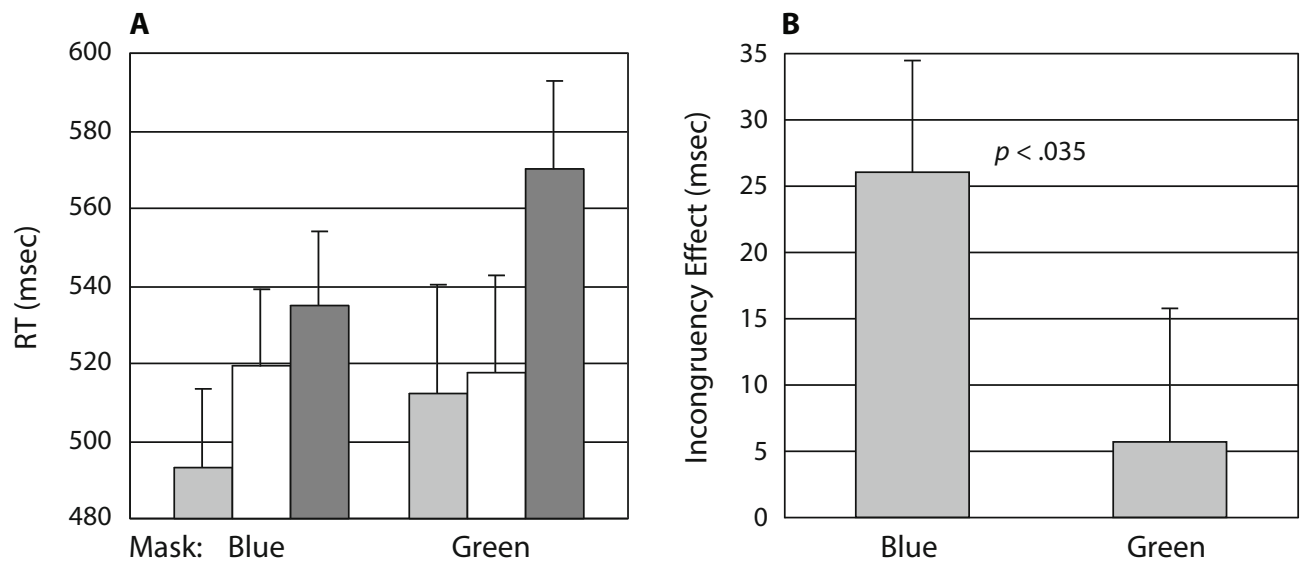

Figure 2. Mean choice reaction times (CRTs) to the blue and green masks when the primes were rendered invisible in the 0/53 (prime invisible) condition; RTs for congruent, white, and incongruent prime-mask pairings are shown by the light gray, white, and dark gray bars, respectively (A). Incongruency effects, the increase in CRTs to the white prime relative to CRTs in the congruent condition, for both blue and green annuli. Vertical bars indicate $1 \operatorname{SEM}(\mathrm{B})$. 
that for both the blue and the green annuli, CRTs were significantly faster in the congruent than in the incongruent condition $[t(13) \geq 5.28, p<.0001$, for both annuli]. Moreover, the overall mean RTs for the blue and green masks were 515.7 and $533.2 \mathrm{msec}$, respectively $[t(41)=$ 2.53, $p<.015]$. Contributing to the interaction was the fact that the white prime raised the CRT to the blue mask by $26.3 \mathrm{msec}$ relative to the congruent condition $[t(13)=$ $2.99, p<.007$ ], but raised the CRT to the green mask by a much smaller and nonsignificant $5.6 \mathrm{msec}$ relative to the congruent condition $[t(13)=0.542, p>.59]$. Figure $2 \mathrm{~B}$ illustrates this difference (increases in CRT relative to the congruent condition) in terms of incongruency effects for each of the mask colors for the white primes. The incongruency effect of the white prime was significantly larger for the blue than for the green mask $[t(13)=2.00, p<$ $.035]$. Thus, the white disk acted like an incongruent green prime for the blue mask and a congruent green prime for the green mask. Replicating the results reported by Breitmeyer, Ro, and Singhal (2004), this result is consistent with the hypothesis that the unconscious priming effect of the white prime occurs at early, stimulus- or wavelengthdependent levels of cortical processing, rather than at later percept-dependent ones.

Mask CRTs for visible primes (3.5/53). In contrast to these results, when the primes were rendered visible by introducing a 3.5 min arc gap between the prime and mask contours, the white disk tended to act as a neutral prime. A 2 (mask color) $\times 3$ (congruency) repeated measures ANOVA revealed a significant main effect of congruency $[F(2,26)=$ $21.88, p<.001]$. Figure $3 \mathrm{~A}$ shows that for both the mask colors, CRTs were significantly faster in the congruent than in the incongruent condition $[t(13) \geq 3.06, p<.005$, for both mask colors]. Moreover, relative to the congruent condition, the white prime raised the CRT to the blue mask by
$13.5 \mathrm{msec}[t(13)=1.46, p<.085]$ and to the green mask by a slightly larger $14.7 \mathrm{msec}[t(13)=2.24, p<.025]$. Figure $3 \mathrm{~B}$ illustrates the similar action of the white prime, in terms of incongruency effects, for each of the mask colors. The incongruency effects were not significantly different for the blue and the green mask $[t(13)=0.10, p>$ $.46]$. Here, in contrast to the different incongruency effects obtained in the $0 / 53$ (prime invisible) condition, the white disk acted indifferently, i.e., as a neutral prime, for the blue and the green annuli. Hence, this neutral effect of the white prime cannot be explained by stimulus-dependent activity. It is better explained by invoking a later, percept-dependent level of cortical processing. This would be confirmed if, perceptually, the white primes were equally similar to the green and blue primes (see below).

Prime visibility and confusion data. All proportions of correct prime-color identifications are in terms of the total number (126) of trials. In the $0 / 53$ condition, on the assumption that observers were not able to perceive the three colors of the prime disks, their performance should be determined purely by a guessing strategy that yields a chance proportion of .333 correct prime-color identifications. The results obtained in the $0 / 53$ condition showed that the average proportion of correct identification was $.359, .026$ above the expected .333 . While the difference of .026 was significant $[t(13)=3.16, p<.01]$, indicating that the observers may have seen the primes in an average of 3 out of 126 trials, we believe that this number is sufficiently small to rule out significant contaminations of the unconscious priming effects by any conscious priming effects. Should that not have been the case, we could reasonably assume that, to the extent that primes were (occasionally) perceived, there should be a significant positive correlation between the proportion of correctly identified prime colors and the priming effect, defined as the dif-

\section{5/53 (Prime Visible)}
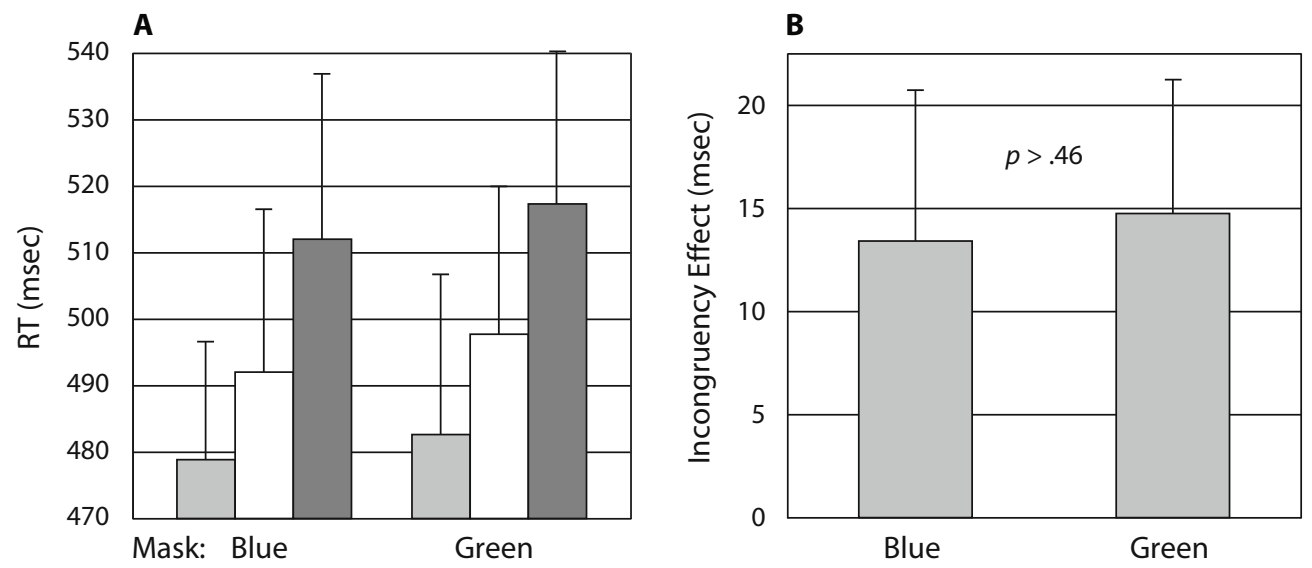

Figure 3. Mean choice reaction times (CRTs) to the blue and green masks when the primes were rendered visible in the $3.5 / 53$ condition. CRTs for congruent, white, and incongruent prime-mask pairings are shown by the light gray, white, and dark gray bars, respectively (A). Incongruency effects, the increase in CRTs to the white prime relative to CRTs in the congruent condition, for both blue and green annuli. Vertical bars indicate $1 S E M(B)$. 
ference between the CRTs obtained in the incongruent and congruent conditions. The correlation across the 14 participants between the priming effect and the proportion of correct prime identifications was .24 , a value not significantly different from $0(p>.40)$.

The results obtained in the $3.5 / 53$ condition showed that, although the correct identification proportion of .515 was far from perfect, it nonetheless differed substantially from the chance performance of $.333[t(13)=5.36, p<.0001]$. Observers' reports indicated that they saw the primes on practically every trial; however, they also reported having had appreciable difficulty perceptually distinguishing their colors. To index the perceptual status of the white relative to the blue and green primes, perceptual color confusions between the white and the blue primes and the white and the green primes were obtained from the respective incorrect color identification responses. The results in Figure 4 reveal that the proportion of white-blue confusions, at .156, was not significantly different from 0.201 , the proportion of white-green confusions $[t(13)=1.97, p>.07]$, indicating that the white prime's perceptual similarity to the blue prime was not different from the similarity to the green prime. (The proportion of blue-green confusions was .129.)

In the $3.5 / 53$ condition, the equal white-green and white-blue color confusions and the equal priming effects of the white target on CRTs to the green and blue mask indicate that here, as expected, equal perceptual similarities produced equal priming effects. Several observers spontaneously reported difficulty in seeing any distinct colors in the primes (as evidenced by their obtaining a correct identification proportion of only .515). One of the authors (BGB) ran himself in the $3.5 / 53$ prime-color identification task and indeed noted that the primes often tended to look whitish (very desaturated), regardless of their actual wavelength composition, whereas, when presented without the mask, they more frequently appeared to have desaturated colors. Recently, Breitmeyer et al. (2006) reported that the visibility of different features of a target - its shape, brightness, etc.- - are not suppressed equally by a metacontrast mask; it is therefore possible that, while the prime's luminance contrast was not suppressed in the $3.5 / 53$ condition thus rendering the primes visible, its color was (at least partially) suppressed, rendering the perception of the already desaturated blue and green primes even more desaturated and thus equally confusable with the white prime.

\section{EXPERIMENT 2}

To address this possibility, we conducted a second experiment in which the prime's visibility was increased by introducing a long SOA at which the prime was clearly visible. Moreover, to assure that the white-blue prime confusions would be greater than the white-green confusions, we conducted the experiment in the same laboratory that yielded the findings reported by Breitmeyer, Ro, and Singhal (2004). ${ }^{1}$

\section{Method}

Participants. Eight students with no knowledge of the purpose of the experiment were selected from the Rice University undergradu- ate student population and served as observers, having given prior voluntary informed consent to their participation. All observers had normal color vision.

Stimuli and Apparatus. The stimuli and apparatus were identical to that used in Breitmeyer, Ro, and Singhal's (2004) study; and, with very minor differences in stimulus spatial dimensions, were like those used in the Experiment 1.

Procedure. The procedure also was identical to that used in Experiment 1 except for the following: In Experiment 2, as in the Breitmeyer, Ro, and Singhal (2004) study, the spatial separation between prime disks serving as primes and the surrounding mask was set to 0 min arc. However, the SOA between the prime and mask was set at $257 \mathrm{msec}$, rendering the colored primes clearly visible. Henceforth we will refer to this as the $0 / 257$ condition.

\section{Results and Discussion}

Mask CRTs for visible primes (0/257). Response errors averaged less than $2.8 \%$. The prime disks, when rendered visible by introducing a $257-\mathrm{msec}$ SOA between the prime and mask, revealed that the white disk again tended to act as a neutral prime. A 2 (mask color) $\times 3$ (congruency) repeated measures ANOVA revealed a significant main effect of congruency $[F(2,14)=11.18, p<.002]$, but neither the main effect of mask color or the interaction between mask color and congruency were significant (both $p \mathrm{~s}>.2$ ). Figure 5A shows that for both mask colors, CRTs were faster in the congruent than in the incongruent condition [blue mask, $t(7)=4.66, p<.003$; green mask, $t(7)=2.23, p=.06]$. Relative to the congruent condition, the white prime raised the CRT to the blue mask by $33.5 \mathrm{msec}[t(7)=2.47, p<.05]$ and to the green mask by a slightly smaller $23.1 \mathrm{msec}[t(7)=2.00, p=.085]$. Figure $5 \mathrm{~B}$ also illustrates the similar action of the white prime, in terms of incongruency effects, for each of the mask colors. The incongruency effects were not significantly different for the blue and the green mask $[t(7)=$ $0.69, p>.51]$. Thus here, in contrast to the different incongruency effects obtained in the $0 / 53$ (prime invisible) condition of Experiment 1, and like the 3.5/53 condition

\section{5/53 (Prime Visible)}

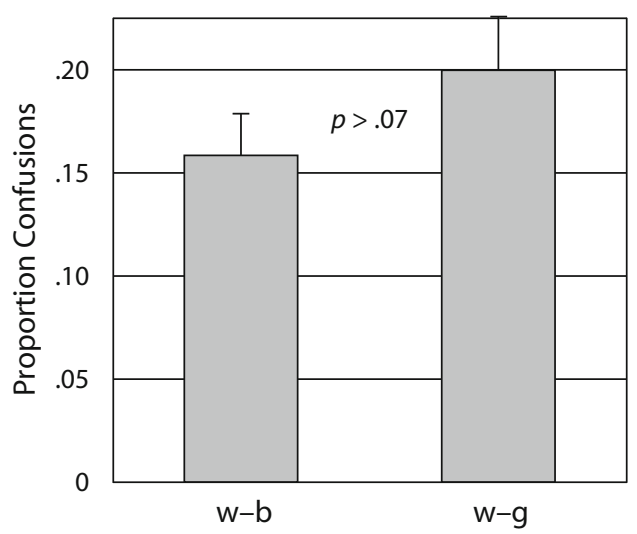

Figure 4. Proportions of perceived color confusions between the white and blue $(w-b)$ primes and the white and green $(w-g)$ primes when primes were rendered visible in the 3.5/53 (prime visible) condition. Vertical bars indicate $1 S E M$. 

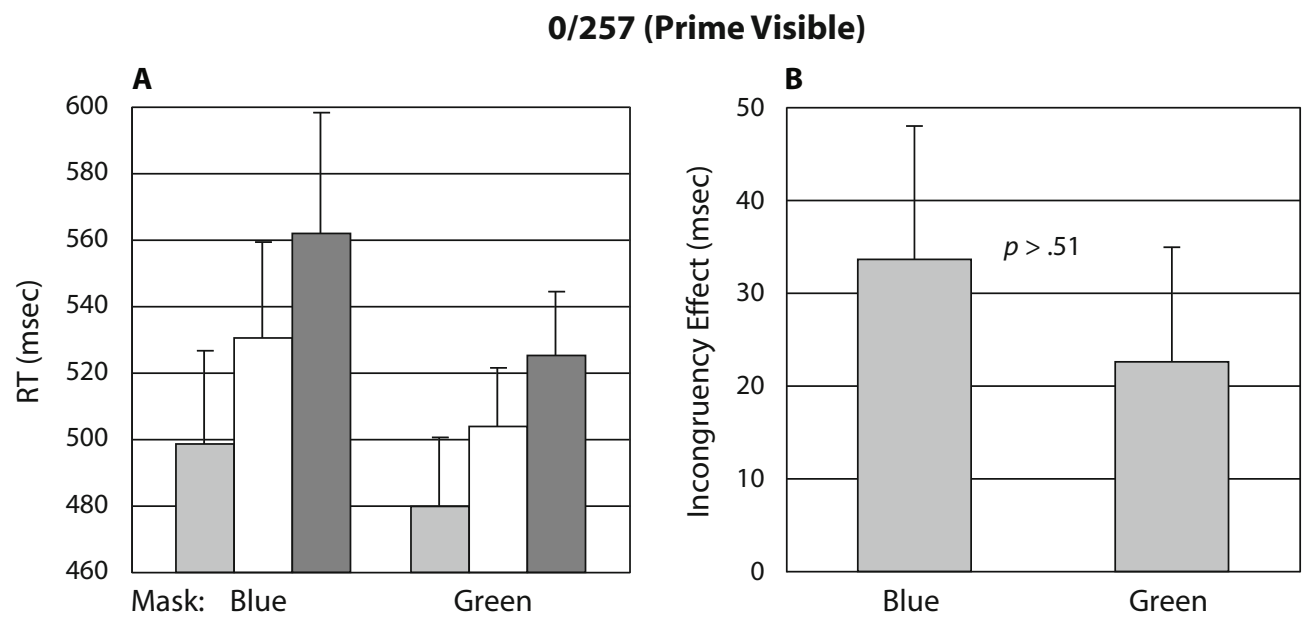

Figure 5. Mean choice reaction times (CRTs) to the blue and green masks when the primes were rendered visible in the $0 / 257$ (prime visible) condition. RTs for congruent, white, and incongruent prime-mask pairings are shown by the light gray, white, and dark gray bars, respectively (A). Incongruency effects, the increase in CRTs to the white prime relative to CRTs in the congruent condition, for both blue and green annuli. Vertical bars indicate 1 SEM (B).

of Experiment 1, the white disk acted indifferently, i.e., as a neutral prime, for the blue and the green annuli.

Prime visibility and confusion data (0/257). Observers reported that they perceived the primes on every trial. The obtained color-confusion results in the $0 / 257$ condition showed that although the correct-identification proportion of .688 was not perfect, it nonetheless differed significantly from the chance proportion of .333 $[t(7)=4.91, p<.002]$. To index the perceptual status of the white prime relative to the blue and green primes, perceptual color confusions between the white and the blue primes and the white and the green primes were obtained from the respective incorrect color identification responses. Unlike the results shown in Figure 4, those in Figure 6 show that-as in the Breitmeyer, Ro, and Singhal (2004) study - the proportion of white-blue confusions, .255, was significantly greater than both the proportions of white-green confusions, .111, and bluegreen confusions, .102 [both $t \mathrm{~s}(7)>2.99, p<.02$ ] . As in the Breitmeyer, Ro, and Singhal study, the proportions of blue-green and white-green confusions did not differ $[t(7)=0.26, p>.40]$. These results indicate that the white prime's perceptual similarity to the blue prime was significantly higher than its similarity to the green prime. Yet, despite this finding, the CRT data shown in Figure 5 reveal that the white prime does not act more like the blue than like the green prime, a result that would be expected if perceptual similarity and percept-dependent neural activities determined the effects of the white prime. Below, we more fully discuss this finding and the insights it provides regarding conscious and unconscious processing.

\section{GENERAL DISCUSSION}

We compared the priming effects of green, blue, and white primes on CRTs to subsequently presented green and blue masks under a condition in which the primes' visibilities were suppressed by masks to priming effects obtained in two conditions in which the primes' visibilities were left partially or totally intact despite the aftercoming mask. The first, 0/53 condition (Experiment 1) replicated prior findings reported by Breitmeyer, Öğmen, and Chen (2004) and Breitmeyer, Ro, and Singhal (2004), showing that the invisible white prime produced greater incongruency effects for the blue and green masks respectively, and thus acted more like the invisible green than the invisible blue prime. Moreover, Breitmeyer, Ro, and Singhal's results indicated that this similarity between the white and green primes is most likely the result of visual processes responsive to the primes' objectively specifiable wavelength properties rather than to their subjectively perceived (color/hue) properties.

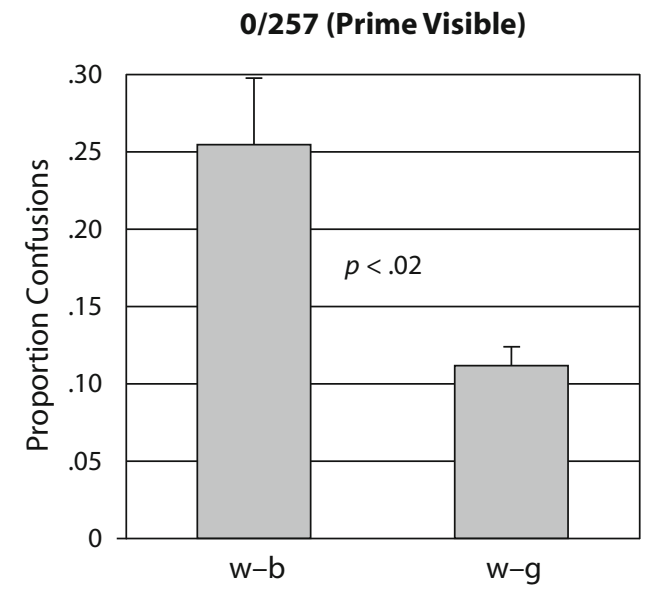

Figure 6. Proportions of perceived color confusions between the white and blue $(w-b)$ primes and the white and green $(w-g)$ primes when primes were rendered visible in the $0 / 257$ (prime visible) condition. Vertical bars indicate $1 S E M$. 
Since the experimental task required observers to use an action plan to differentially respond to the clearly visible blue and green masks, one would expect that the differences between the wavelength-dependent activations produced by the blue and green stimuli would provide the basis for generating the differential responses. Moreover, such wavelength properties ought to be expressible in terms of corresponding cone contrast values. Note that Table 2 displays the three primes' LW and MW contrasts as being nearly identical, thus yielding differential contrast values nearly equal to zero (all contrast differences among the three primes are .008 or less). For that reason, it is unlikely that a wavelength-dependent code that differentiates between the three primes is based on differences between LW or MW cone contrasts. In comparison with these low contrast differences, note that what differentiates the green prime's (and mask's) relative to the blue prime's (and mask's) SW cone contrast is a fairly large reduction of .19. In turn, what differentiates the white prime's from the blue prime's SW cone contrast is a somewhat smaller but still also a relatively large reduction of .092. The fact that both the green and the white primes' SW contrasts are reduced relative to that of the blue prime - or, vice versa, that both the blue the white primes' SW contrasts are raised relative to that of the blue prime - could be a basis for the white prime behaving more like the green prime than the blue prime, at unconscious levels of processing. This would be consistent with the unconscious colorpriming effect occurring at early wavelength-dependent levels of processing instead of at later percept-dependent levels. Since this would be the case on every trial when a prime was fully masked, it follows that, averaged across a large majority of such trials, the visibly suppressed white prime acts as a green (MW) prime more strongly than it acts as a blue (SW) one.

One could, of course, argue that the masked primes, although invisible, somehow activate percept-dependent levels of processing at which white is more easily confused with green than with blue, in which case, as indicated by the color confusion results obtained in the $0 / 257$ condition of Experiment 2 (as well as in Breitmeyer, Ro, \& Singhal's 2004 study), at a still later percept-dependent level this confusion would have to be reversed, revealing a color-coding strategy in the visual system that would be much more complicated and puzzling than one in which a percept-dependent coding, once initiated, was not reversed. Although we cannot rule out such a complicated process, for reasons of conceptual economy we adopt, in the case of the $0 / 53$ condition, the simper, stimulusdependent process.

In contrast, the other two conditions, 3.5/53 (Experiment 1) and 0/257 (Experiment 2), which rendered the primes visible, yielded results consistent with the white disk acting as a neutral prime, since its incongruency effects were equal for the green and blue masks. This indicates that the effects of the perceived colored primes in a block of trials did not occur exclusively at either the early stimulus-dependent or at the later percept-dependent level of cortical color processing. Although these priming effects may occur in part at percept-dependent levels, we propose that they also occur at earlier chromatic processing levels that include stimulus-dependent ones.

The neutral effects of the white prime could be explained as follows. Especially in the 3.5/53 condition, one possibility is that the priming effects across trials may have been determined by a mix of trials generating stimulusdependent processes (when the color of the primes was masked) and of trials generating percept-dependent processes (when the prime colors were not masked). Although the primes were perceivable on practically every trial, because of the short SOA of $53 \mathrm{msec}$ on a substantial number of trials the processing of the prime's chromatic properties may still have been at the stimulus-dependent level when the responses to the following mask were being programmed; that is, on these trials the prime's visual processing at the percept-dependent level and hence the perception of the prime occurred after the response to the mask was already determined. While this would not affect the total priming effect-i.e., the differences between the incongruent and congruent CRTs - the percept-dependent and stimulus-dependent effects of the white prime would cancel each other out across trials, thus rendering its priming effect equal for the blue and the green masks. ${ }^{2}$

Moreover, the phenomenology of the 3.5/53 and the $0 / 257$ condition indicates that there may have been differences in the percept-dependent activations produced by the primes. Recall that the primes in the $3.5 / 53$ condition tended to appear very desaturated (nearly white), while their colors were more clearly distinguishable in the 0/257 condition; in other words, they should have acted more strongly as a neutral prime in the $3.5 / 53$ condition than in the $0 / 257$ one. If so, the differential priming effects of the white prime on the blue- versus the greenmask CRTs in the 3.5/53 condition should not correlate strongly with differential color confusions of the white prime with the blue versus the green prime. ${ }^{3}$ To test this, we computed for each of the 14 observers the difference between the incongruency effects produced by the white prime for the blue versus the green masks. This difference value is therefore related to the strength of the "green" or "blue" effect of the white prime, with large positive differences indicating a stronger green than blue effect (with white acting more like an incongruent prime for the blue mask), zero indicating no differential effects of the white prime (with white acting as neutral prime), and large negative differences indicating stronger blue than green effects (with white acting more like an incongruent prime for the green mask). We also computed for each observer a similar difference between the proportions of his or her confusion between white and green and white and blue. These difference values are therefore directly related to the perceptual similarity of the white prime to the green and blue primes, with larger positive differences indicating a stronger similarity of white to green than to blue, zero indicating equal similarities of white to green and to blue, and larger negative values indicating a stronger similarity of white to blue than to green. Across the 14 observers in the $3.5 / 53$ condition, the correlation between these two indices was .003 ( $p>.495$, one-tailed). This indicates that the effects of the white prime on the CRTs 
of the blue and green masks on the one hand, and the color similarities of the white prime to a green or blue one on the other hand, were not systematically related. A similar analysis was performed on the results of the 8 observers in the $0 / 257$ condition, in which the prime colors were more visible. Here the correlation between the same indices was .344 ( $p>.202$, one-tailed). Although this correlation was not statistically significant, it tends to suggest a systematic relation between the differential incongruency effects of the white prime on the CRTs of the blue and green mask and its differential perceptual similarity to the blue and green primes. In future research, this more subtle measure, obtained with a larger number of observers, may reveal the existence of a statistically significant percept/ color-dependent priming effect, but most likely only when the prime is rendered entirely visible by having the mask follow at a large SOA.

\section{AUTHOR NOTE}

B.G.B. is associated with the Department of Psychology and the Center for Neuro-Engineering and Cognitive Science at the University of Houston. T.R. is associated with the Department of Psychology at Rice University. H.O. is associated with the Department of Electrical and Computer Engineering and the Center for Neuro-Engineering and Cognitive Science at the University of Houston. S.T. is associated with the Department of Philosophy at the University of Houston. This work was supported by NSF Grant BCS-0114533 and NIH Grant R01-MH49892. We thank Jennifer Boyer for assistance with the data collection for Experiment 2. We also thank the three reviewers, Simon Cropper, Michael Snodgrass, and Thomas Schmidt, for suggesting very helpful substantive and stylistic improvements to our manuscript. Correspondence concerning this article should be addressed to B. G. Breitmeyer, Department of Psychology, University of Houston, Houston, TX 77204-5022 (e-mail: brunob@uh.edu).

\section{REFERENCES}

Ansorge, U., Klotz, W., \& Neumann, O. (1998). Manual and verbal responses to completely masked (unreportable) stimuli: Exploring some conditions for the metacontrast dissociation. Perception, 27, 1177-1189.

BreitMeyer, B. G. (1984). Visual masking: An integrative approach. New York: Oxford University Press.

Breitmeyer, B. G., Kafaligönül, H., ÖĞmen, H., Mardon, L., TodD, S., \& Ziegler, R. (2006). Meta- and paracontrast reveal differences between contour- and brightness-processing mechanisms. Vision Research, 46, 2645-2658.

Breitmeyer, B. G., ÖĞMEN, H., \& Chen, J. (2004). Unconscious priming by color and form: Different processes and levels. Consciousness \& Cognition, 13, 138-157.

Breitmeyer, B. G., Ro, T., \& Singhal, N. S. (2004). Unconscious color priming occurs at stimulus- not percept-dependent levels of visual processing. Psychological Science, 15, 198-202.

CRICK, F., \& КосH, C. (1998). Consciousness and neuroscience. Cerebral Cortex, 8, 97-107. de Gelder, B., de Haan, E. H. F., \& Heywood, C. (Eds.). (2001). Out of mind. New York: Oxford University Press.

Dolan, R. J. (2002). Emotion, cognition, and behavior. Science, 298, 1191-1194.

Esteves, F., Parra, C., Dimberg, V., \& Ohman, A. (1994). Nonconscious associative learning: Pavlovian conditioning of skin conductance responses to masked fear-relevant facial stimuli. Psychophysiology, 31, 375-385.

KinLSTROM, J. F. (1996). Perception without awareness of what is perceived, learning without awareness of what is learned. In V. Velman (Ed.), The science of consciousness (pp. 23-46). London: Routledge.

Klotz, W., \& WolfF, P. (1995). The effect of a masked stimulus on the response to the masking stimulus. Psychological Research, 58, 92-101.

Kreiman, G., Fried, I., \& Koch, C. (2002). Single-neuron correlates of subjective vision in the human medial temporal lobe. Proceedings of the National Academy of Sciences, 99, 8378-8383.

LeOPold, D. A., \& Logothetis, N. K. (1996). Activity changes in early visual cortex reflect monkey's percepts during binocular rivalry. $\mathrm{Na}$ ture, 379, 549-553.

Metzinger, T. (2000). Neural correlates of consciousness. Cambridge, MA: MIT Press.

Milner, A. D., \& Goodale, M. A. (1995). The visual brain in action. Oxford: Oxford University Press.

Morris, J., \& Dolan, R. (2001). The amygdala and unconscious fear processing. In B. de Gelder, E. H. F. de Haan, \& C. Heywood (Eds.), Out of mind (pp. 185-204). New York: Oxford University Press.

Neumann, O., \& Klotz, W. (1994). Motor responses to nonreportable, masked stimuli: Where is the limit of direct parameter specification? In C. Umiltà \& M. Moscovitch (Eds.), Attention \& performance XV (pp. 123-150). Cambridge, MA: MIT Press.

Schmidt, T. (2000). Visual perception without awareness: Priming responses by color. In T. Metzinger (Ed.), Neural correlates of consciousness (pp. 157-169). Cambridge, MA: MIT Press.

Schmidt, T. (2002). The finger in flight: Real-time motor control by visually masked color stimuli. Psychological Science, 13, 112-118.

Vorberg, D., Mattler, U., Heinecke, A., Schmidt, T., \& Schwarzвасн, J. (2003). Different time course for visual perception and action priming. Proceedings of the National Academy of Sciences, 100, 6275-6280.

Weiskrantz, L. (1997). Consciousness lost and found: A neuropsychological exploration. New York: Oxford University Press.

ZEKI, S. (1997). The color and motion systems as guides to conscious visual perception. In K. S. Rockland, J. H. Kaas, \& A. Peters (Eds.), Cerebral cortex: Vol. 12. Extrastriate cortex of primates (pp. 777809). New York: Plenum.

\section{NOTES}

1. Experiment 1 was conducted in B.G.B.'s laboratory at the University of Houston; Experiment 2 in T.R.'s lab at Rice University.

2. Michael Snodgrass suggested this alternative explanation to us.

3. We again thank Michael Snodgrass for suggesting the following way of analyzing our results.

(Manuscript received October 21, 2004; revision accepted for publication September 6, 2006.) 